\title{
Implementation of Pedestrian Path Navigation Algorithm Based on "We-Map" Platform
}

\author{
Jian Zhang ${ }^{\text {a }}$, Haowen Yan ${ }^{\text {a, * }}$ \\ ${ }^{a}$ Faculty of Geomatics, Lanzhou Jiaotong University,Lanzhou,China
}

Keywords: we media, we-map, landmark, pedestrian navigation, path guidance

\begin{abstract}
:
In order to adapt to the information needs of the we media era, Haowen Yan and other scholars proposed a "grassroots" map for the civilian population---"We-Map". The mathematical foundations such as accuracy are not high, and the producers do not need to undergo strict professional training. Map users can participate in map production at any time, and can easily and quickly communicate and apply on personal electronic devices (such as computers and mobile phones) ${ }^{[1]}$. In the We-Map platform, it uses the Google Map SDK and XML technologies to implement the basic functions of the mobile platform-based We-Map application, including map editing (copy, rotate, zoom, pan, line drawing, coloring, etc.), path navigation, Real-time location, information query and WeMap storage, transmission and distribution. In this paper, we study and implement a landmark-based pedestrian path navigation algorithm, which is added to the We-Map platform for auxiliary navigation of pedestrian paths.
\end{abstract}

At present, pedestrian navigation software based on mobile terminals mainly inherits the car navigation mode and concept, and uses a technical solution similar to that of car navigation ${ }^{[2]}$, that is, navigation tasks according to satellite GPS positioning and electronic compass and magnetometer orientation. However, the pedestrian path is more autonomous, more selective, and more random. This makes the use of traditional navigation ideas not suitable for pedestrian navigation, so this article will introduce the concept of "landmarks" in pedestrian navigation. Landmarks can help pedestrians with normal vision to complete path-finding decisions, enhance pedestrians' trust in the path and space security, and reduce cognitive burden ${ }^{[3]}$.

The pedestrian navigation system studied in this paper consists of electronic maps, spatial positioning, path planning and path guidance. The key technologies and specific functions of each module are introduced below.

In the electronic map and spatial positioning module, using Google map as the carrier, and based on the satellite positioning, combined with the surrounding landmarks to confirm, to achieve accurate positioning, a digital model that conforms to pedestrian spatial cognition is established. In the path planning module, the user can use his own drawing or downloading the map path that other people have drawn. In the drawing process of the map, a typical and identifiable landmark can be added as a reference to increase the pedestrian's credibility to the path. Through the form of such a public source map, it is possible to realize the self-planning of the pedestrians according to the self-requirement path, to achieve diversity, and to meet various needs of users. In the path guiding module, the navigation is performed according to the planned route of the user, and the landmark element is added in the description sentence to reduce the user's judgment error, and assist the user to quickly and accurately reach the destination along the pre-planned path.

Path-guided process implementation:

1. Establish a landmark database.

2. According to the spatial relationship of landmarks, establish a position description statement that conforms to pedestrian cognition. The landmarks are classified according to nodes and roads and used for different navigation statements.

3. According to the planned directed route, extract the landmarks on the way and establish the orientation information between the landmarks for the overall orientation of the route. This generates the statement "Go straight to the ... (landmark name)".

4. According to the planned directed route, each inflection point is extracted for finding significant landmarks around the decision point, and these landmarks will be used for the discrimination of the pedestrian's route. The statement is "Left / Right Turn will see ... (landmark name)".

5. Use the road as the center line to establish a buffer zone (the specific threshold is set according to the actual situation (road width, pedestrian line of sight, lighting conditions, etc.)). According to the planned route that has 
been planned, look for the landmarks that have passed along the way to generate the sentence "Through ... (landmark name)".

6. In the navigation, update the location information in real time, and use this as a new starting point, and set "Where am I?" to quickly help pedestrians to identify their position in real time and prevent mistakes.

In general, this article adds the concept of landmarks on the basis of We-Map. On one hand, everyone can participate in the process of mapping, and can upload and exchange online and download and use, solve the different requirements of different groups of people today, and fully consider the various needs of pedestrians; On the other hand, in the pedestrian navigation function, compared with the traditional navigation, the use of landmarks makes the navigation clearer and more accurate, more in line with the cognition of various people, and makes the map and navigation serve more people.

\section{References:}

[1] Yan Haowen, Zhang Liming, Du Ping, Liu Tao, Wang Zhonghui, Yang Weifang. We-Map: A New Type of Map in the Era of We Media[J]. Journal of Geomatics Science and Technology,2016,33(05):520-523.

[2] Millonig A , Schechtner K . Developing Landmark-Based Pedestrian-Navigation Systems[J]. IEEE Transactions on Intelligent Transportation Systems, 2007, 8(1):43-49.

[3] ZHANG Xing, LI Qingquan, FANG Zhixiang, HUANG Ling. Landmark and Branch-based Pedestrian Route Complexity and Selection Algorithm. GEOMATICS AND INFORMATION SCIENCE OF WUHAN UNIVERS, 2013, 38(19):1239-1242. 